\title{
Der aktuelle Perspektivenstreit in der Stadtsoziologie
}

\section{Einleitung}

Am Beginn der Kontroverse steht eine simple Frage: Kann »Stadt « ein Gegenstand soziologischer Analysen und Theoriebildung sein? Um die Beantwortung dieser Frage streitet man sich in der deutschen Stadtsoziologie seit Jahrzehnten. ${ }^{1}$ Heute halten sie manche für "gelöst " oder » obsolet geworden ", ${ }^{2}$ andere für » umstrittener denn je «. ${ }^{3}$ Einig ist man sich darüber, dass es bei der Frage nach dem sozialwissenschaftlichen Gegenstand "Stadt " »ums Ganze «" geht. Sie führt in den Kern des stadtsoziologischen Selbstverständnisses, ${ }^{5}$ sind mit ihr doch nicht weniger brisante Folgefragen verbunden: Kann die Stadt als » eine eigenständige Ursache gesellschaftlicher Entwicklungen ${ }^{6}{ }^{6}$ verstanden werden? Was sonst, wenn nicht »die Stadt ", sollte im Fokus der Teildisziplin Stadtsoziologie stehen? Was gewinnt und was verliert man, wenn man das Städtische als eigenständigen Modus der Vergesellschaftung begreift? Und schließlich: Wie und worüber soll man forschen?

In den letzten zehn Jahren ist in der deutschen Stadtsoziologie um diesen Fragenkomplex eine lebhafte, bisweilen auch polemisch geführte Debatte entstanden, um die es im Folgenden gehen soll. Grundsätzlich lassen sich zwei Positionen unterscheiden: Der erste - »kritische « -Ansatz wird vor allem durch Hartmut Häußermann und Walter Siebel ${ }^{7}$ vertreten, für die die Stadt in Europa zuletzt in einer bestimmten Epoche - nämlich im europäischen Mittelalter - zum »historischen Subjekt ${ }^{8}{ }^{8}$ wurde. Mit fortschreitender Industrialisierung sei der die Gesellschaftsentwicklung antreibende Gegensatz zwischen Stadt und Land im 19. Jahrhundert verschwunden. Die Stadt sei deshalb heute kein abgrenzbarer Gegenstand mehr, und Stadtsoziologie müsse deshalb Gesellschaftsanalyse in der Stadt sein.

Den zweiten - »eigenlogischen « - Ansatz haben Helmuth Berking und Martina Löw vorgelegt. ${ }^{9}$ Sie gehen von einem an Louis Wirth orientierten universellen Stadt-

1 Vgl. exemplarisch Häußermann, Siebel 1978; Krämer-Badoni 1991.

2 Krämer-Badoni 2011, S. 70.

3 Berking, Löw 2005, S. 9.

4 Krämer-Badoni 2011, S. 70.

5 Vgl. dazu jüngst Herrmann et al. 2011.

6 Häußermann, Siebel 2004, S. 89.

7 Häußermann, Siebel 1978; Häußermann, Siebel 2004.

8 Häußermann, Siebel 2004, S. 90.

9 Berking, Löw 2008 b. 
begriff aus, den sie um wissens- und raumsoziologische Elemente erweitern. Berking und Löw verstehen Stadt als »raumstrukturelle Form ", die Dichte und Heterogenität organisiert. ${ }^{10}$ In jeder Stadt verlaufe das (etwas) anders. Das Resultat städtischer Verdichtungs- und Heterogenisierungsprozesse seien spezifische und abgrenzbare Sinnhorizonte des Handelns, ${ }^{11}$ die sich über vor Ort eingespielte Prozesse der Habitualisierung, Institutionalisierung und Materialisierung stabilisierten. Auf diese Weise entstünden »städtische Eigenlogiken «.

Frappant ist, dass die ProtagonistInnen der hier kurz umrissenen Forschungsansätze bislang kaum direkt aufeinander reagiert haben. ${ }^{12}$ Zwar fanden in den vergangenen Jahren einige Diskussionen statt, doch verliefen diese ausschließlich in geschlossenen Veranstaltungsformaten, die zu keiner gemeinsamen Publikation führten. Aus diesem Grund wurde von den VerfasserInnen dieses Beitrags auf dem 36. Kongress der Deutschen Gesellschaft für Soziologie im Oktober 2012 eine Adhoc-Gruppe zum aktuellen Perspektivenstreit in der Stadtsoziologie veranstaltet. Ihr Ziel war es, den schwelenden, die Forschung teilweise hemmenden Konflikt um den Gegenstandsbereich "Stadt " in der Stadtsoziologie einer öffentlichen Diskussion zugänglich zu machen und einen Austausch von Argumenten anzustoßen. Die ausgearbeiteten Vorträge von Helmuth Berking (zum Eigenlogik-Ansatz) und Walter Siebel (zur Kritik des Eigenlogik-Ansatzes aus der Perspektive der kritischen Stadtsoziologie) sind in dieser Ausgabe des Leviathan versammelt.

Im Folgenden werden in der Absicht, strukturiert in den bisherigen Stand der Debatte um "Stadt " und "Eigenlogik « einzuführen, die beiden konkurrierenden Positionen entlang ihres Stadt-, Raum- und Gesellschaftsverständnisses rekonstruiert und die sich daraus ergebenden Implikationen für die soziologische Beschäftigung mit Städten aufgezeigt. Bei der Darstellung des Eigenlogik-Ansatzes werden aufgrund des einführenden Beitrags von Helmuth Berking in diesem Heft schwerpunktmäßig die Theorietraditionen herausgearbeitet, die dieser Perspektive zugrunde liegen. Danach skizzieren wir die wichtigsten Anschlussdebatten an den genannten Perspektivenstreit in der Stadtsoziologie. Sie beziehen sich zum einen auf kritische Einwände und offene Fragen gegenüber dem Eigenlogik-Konzept und zum anderen auf das Aufgreifen desselben in den Nachbardisziplinen. Abschließend möchten wir die Herausforderungen, vor denen die Stadtsoziologie aktuell steht, umreißen und auf die Relevanz des hier vorgestellten Perspektivenstreits für die Soziologie insgesamt hinführen.

10 Berking 2008, S. $19 \mathrm{ff}$.

11 Löw 2011, S. 57.

12 Für eine Ausnahme vgl. Häußermann 2011. 


\section{Positionen}

\subsection{Kritische Stadtsoziologie}

Im Jahr 1978 erschien im Leviathan ein mit »Thesen zur Soziologie der Stadt « überschriebener programmatischer Beitrag von Hartmut Häußermann und Walter Siebel, ${ }^{13}$ der rückblickend als Gründungsdokument einer »kritischen Stadtsoziologie « im deutschen Sprachraum betrachtet werden kann. In ihm nahmen Häußermann und Siebel eine Neubestimmung des Gegenstandsbereichs und der Aufgaben der Stadtsoziologie vor, die die beiden Autoren in den folgenden Dekaden in verschiedenen Zeitschriften- und Buchpublikationen, insbesondere aber in ihrem 2004 erschienenen Lehrbuch »Stadtsoziologie. Eine Einführung «14 weiter ausarbeiten und pointieren sollten. Den Grundannahmen dieser kritischen Stadtsoziologie ist die stadtsoziologische Wissensproduktion in den letzten Jahrzehnten weitgehend gefolgt.

Im Zentrum von Häußermanns und Siebels Überlegungen zu Gegenstandsbereich und Aufgaben der Stadtsoziologie steht eine an den historischen Materialismus ${ }^{15}$ angelehnte Theorie sozialen Wandels. Träger des sozialen Wandels sind Gesellschaften in historisch spezifischen, aufeinander folgenden Gesellschaftsformationen. Entsprechend wird sozialer Wandel von Häußermann und Siebel teleologisch gedeutet und als gesellschaftliche Entwicklung verstanden. ${ }^{16}$

Vorangetrieben wird der soziale Wandel durch Klassengegensätze. Häußermann und Siebel legen dar, dass die Klassengegensätze in Europa bis ins Zeitalter des durchgesetzten Kapitalismus mit dem Gegensatz von »Stadt " und »Land « zusammenfielen. ${ }^{17}$ Während im Mittelalter in den Städten das Bürgertum, das Handwerk sowie Handel und Gewerbe zu erblühen begannen, war das Land durch agrarische Produktions- und feudale Herrschaftsstrukturen gekennzeichnet. Mit der Industrialisierung löste sich der Gegensatz zwischen Stadt und Land allmählich auf »zu einem Mehr-oder-Weniger vom Selben «: ${ }^{18}$ Die feudalen Strukturen auf dem Land verschwanden, und die »kapitalistische Durchorganisation aller Arbeitsbereiche « ließ »die vormals >städtischen` sozialen Beziehungen ubiquitär werden «. ${ }^{19}$ Durch den Zuzug von Landarbeitern in die Stadt etablierte sich im Verlauf des 19. Jahrhunderts in den Städten ein Proletariat - mit dem Ergebnis, dass der bisher den sozialen Wandel tragende Gegensatz zwischen Stadt und Land in einen »Widerspruch zwischen Kapital und Arbeit innerhalb der Stadt und des Systems der Städte ${ }^{20}$ umschlug. In der zweiten Hälfte des 20 . Jahrhunderts wurden im Zuge

13 Häußermann, Siebel 1978.

14 Häußermann, Siebel 2004.

15 Marx, Engels 1969 [1932].

16 Vgl. Häußermann, Siebel 1978; Häußermann, Siebel 2004, S. 89 ff.

17 Vgl. Häußermann, Siebel 2004, S. 90 f.; Häußermann, Siebel 1987, S. 91 ff.

18 Häußermann, Siebel 1978, S. 486.

19 Ebd., S. 496.

20 Häußermann, Siebel 2004, S. 91.

Leviathan, 41. Jg., 2/2013 
der Herausbildung nationaler Wachstums- und Konjunkturpolitiken die räumliche Planung, die Organisation der Reproduktion sowie sozialpolitische Interventionen zentralisiert und an den bürokratischen Nationalstaat delegiert. Dies geschah in dem Bestreben, die Folgeprobleme kapitalistischer Entwicklung - den sich verschärfenden Gegensatz zwischen Kapital und Arbeit in den Städten - abzufedern. Seit den 1970er Jahren aber wurden die finanziellen Zuweisungen an die Städte massiv beschnitten, sodass sich die kommunale Steuerungskapazität immer weiter verringerte. Hieraus folgern Häußermann und Siebel: »Im Unterschied zu antiken und mittelalterlichen Stadtstaaten ist heute Stadt weder politisch noch ökonomisch oder gar militärisch eine selbständige Einheit. Die Einbindung der kommunalen Administration in das nationale Rechtssystem und in die Politik von Bund und Ländern sowie die Integration der ortsansässigen Produktion in national und international verflochtene Märkte haben auch einer lokal gebundenen Sozial- und Kommunikationsstruktur die Basis entzogen «.21

Auf die sich um die Wende vom 19. zum 20. Jahrhundert herausbildende Stadtsoziologie hatte das Verschwinden der Polarität von Stadt und Land und die Zentralisierung und Verstaatlichung vormals kommunaler Aufgabenbereiche weitgehende Auswirkungen. Laut Häußermann und Siebel verlor sie ihren Gegenstand: "Mit der Auflösung eines durch Unterschiede der Produktion und des Überbaus gekennzeichneten Gegensatzes von Stadt und Land kann Stadtsoziologie sich nicht (mehr) auf einen sozialen Tatbestand als ihren eigenen Gegenstand berufen «.22 In soziologischen Untersuchungen könne Stadt folglich nur noch als » physisches Phänomen ${ }^{23}$, nicht aber als » eigenständige Ursache gesellschaftlicher Entwicklungen « fungieren. ${ }^{24}$ Als neuen Gegenstandsbereich der Stadtsoziologie im durchgesetzten Kapitalismus identifizierten Häußermann und Siebel »Gesellschaft «. Für sie ist die Untersuchung von Städten seit dem ausgehenden 19. Jahrhundert »in Wirklichkeit die Untersuchung der >modernen $>$ (= industriellen) Gesellschaft, die Stadt also nur der Ort, an dem die Gesellschaft in ihrer Struktur und ihren Konflikten erscheint $\ll .25$

Entsprechend verwehren sie sich gegen Ansätze, » in denen die Großstadt oder die räumliche Verteilung sozialer Gruppen für die politischen und sozialen Konsequenzen einer kapitalistisch organisierten Industrialisierung verantwortlich gemacht werden «. ${ }^{26}$ Dies richtet sich vor allem gegen Forschungsarbeiten, die räumliche Strukturen und ihre Veränderungen als eigenständige Ursache sozialer Phänomene fassen. Ein solches Vorgehen betrachten Häußermann und Siebel als eine eklatante »Verwechslung von Ursache und Wirkung, von zugrundeliegender Struktur und Erscheinungsform « und als »Geburtsfehler jeder Soziologie der Stadt, seit es Stadt

21 Häußermann, Siebel 1978, S. 485 f.

22 Ebd., S. 486.

23 Ebd.

24 Häußermann, Siebel 2004, S. 89.

25 Häußermann, Siebel 1978, S. 486.

26 Ebd. 
als lokale Identität nicht mehr gibt «. ${ }^{27}$ Für sie sind die räumlichen und sozialen Strukturen der Stadt »integrale Bestandteile des Vergesellschaftungsprozesses « was für sie bedeutet, dass »weder räumliche Struktur noch die Gemeinde eigenständige Gegenstände soziologischer Forschung sein können «. ${ }^{28}$ Den Ansatz von Louis Wirth, ${ }^{29}$ »eine lineare Kausalität zwischen Größe, Dichte und Heterogenität der Bevölkerung (unabhängige, erklärende Variable) und der Lebensweise der Städter als deren Folge (abhängige Variable) « ${ }^{30}$ herzustellen, brandmarken Häußermann und Siebel als »Rückfall in die simplifizierenden Argumentationsmuster der konservativen Großstadtkritik, die die physischen Tatsachen der Stadt für die sozialen Probleme der kapitalistischen Industriegesellschaft verantwortlich gemacht hatte " ${ }^{31}$ Stattdessen sei das, was in den großen Städten geschehe, »eine Konsequenz sozialer Veränderungen, hauptsächlich der kapitalistisch organisierten Industrialisierung «. ${ }^{32}$ Infolgedessen rufen Häußermann und Siebel die Stadtsoziologie dazu auf, »die vorfindlichen städtischen Strukturen selber zu diesen Konsequenzen $\aleph^{33} \mathrm{zu}$ zählen, also den Zusammenhang zwischen der gesellschaftlichen (kapitalistischen) Entwicklung und der Entwicklung der Stadt zu thematisieren. Damit laute die zentrale Frage der Stadtsoziologie nicht mehr » was heißt städtisches Leben im Vergleich zum Leben auf dem Lande, sondern: was bedeutet Stadtentwicklung für die Entwicklung des Klassenverhältnisses? «. 34

Vor diesem Hintergrund werden nun die Aufgaben einer kritischen Stadtsoziologie definiert. Laut Häußermann und Siebel muss eine kritische Stadtsoziologie erstens Ideologiekritik betreiben, also vorherrschende Problemdefinitionen und theoretische Positionen der Wissenschaft hinterfragen. ${ }^{35}$ So problematisieren sie die bisherige Tendenz der Stadtsoziologie, allein Probleme des Bürgertums als »relevante « städtische Probleme und allein die Kultur der Bourgeoisie als »urbane « Kultur zu betrachten. Demgegenüber würden proletarische Lebensverhältnisse bei der soziologischen Theoriebildung zumeist vernachlässigt. ${ }^{36}$ Für Häußermann und Siebel ist die Stadt im Zeitalter des Kapitalismus nicht nur Ort, sondern, indem die Stadt sich immer mehr entlang »den Bedürfnissen von Industrie- und Handelskapital wandelt «, auch » Medium der Ausbeutung «. ${ }^{37}$ Insofern lautet die zweite Aufgabe der kritischen Stadtsoziologie, die Situation und die »Entwicklung des Niveaus der

27 Ebd., S. 487.

28 Ebd., S. 488; vgl. Siebel 2012.

29 Wirth 1974 [1938].

30 Häußermann, Siebel 2004, S. 94.

31 Ebd., S. 93; vgl. Krämer-Badoni 1991.

32 Häußermann, Siebel 2004, S. 96.

33 Häußermann, Siebel 1978, S. 486.

34 Ebd., S. 496.

35 Ebd., S. 494.

36 Vgl. ebd., S. 495.

37 Ebd., S. 497. 
Reproduktionsbedingungen der verschiedenen Schichten der Lohnabhängigen « 38 in der Stadt zu beschreiben und ihre räumlichen Ausprägungen und Wirkungen zu analysieren. Die dritte Aufgabe liegt laut Häußermann und Siebel in der Analyse der Krise einer staatlich regulierten Stadtpolitik, die zu »Privatisierung, Monetarisierung und Individualisierung der Reproduktion ${ }^{39}$ und somit zu enormen sozialräumlichen Polarisierungen geführt habe, und damit in der Analyse »der konkreten Erfahrung, der unmittelbaren, kumulierten Betroffenheit von kapitalistischen Entwicklungsprozessen ${ }^{4}{ }^{40}$

Die Aufforderung an die kritische Stadtsoziologie, Ideologiekritik zu betreiben und soziale Ungleichheiten vor Ort zu beschreiben und in die gesellschaftliche Entwicklung einzubetten, ist - nach der oben beschriebenen Absage an die Untersuchung räumlicher Strukturen - auch als eine Absage an die Erforschung kultureller Phänomene wie Verhaltensstile zu verstehen: Die mit einer solchen Forschung einhergehende Grundannahme, dass es »etwas typisch Städtisches über alle Klassenund Schichtungsgrenzen hinweg gibt ", gilt Häußermann und Siebel insofern als »unzulässige Vereinfachung ", als dass sich »Lebens- und Verhaltensstil städtischer Bourgeoisie und städtischen Proletariats kaum auf einen sinnvoll gemeinsamen Nenner bringen lassen « werden. ${ }^{41}$

Dennoch - die Stadt ist auch eine Produktivkraft. So, wie sich Kapital und Produktionsstätten in der Stadt konzentrieren, so konzentrieren sich hier auch die lohnabhängig Beschäftigten. Hier manifestiert sich der Gegensatz von Kapital und Arbeit am deutlichsten. ${ }^{42}$ Entsprechend sehen Häußermann und Siebel die Stadt als einen »Ort, an dem aus der Arbeiterklasse san sich eine Klasse >für sich konnte [...] und wo sich durch die räumliche Zusammenballung die Dynamik des Klassenkampfes entfaltet $"{ }^{43}$ Dies bedeutet jedoch nicht, wie die Autoren betonen, dass "Stadt « im Zeitalter des durchgesetzten Kapitalismus als »eigenständiger Faktor, der die soziale Entwicklung beeinflusst « ${ }^{44}$ betrachtet werden kann. Stattdessen erläutern sie: »Die Stadt trägt nichts mehr zur Entwicklung der Gesellschaft bei, sie ist vielmehr aus dieser zu erklären. Damit gibt es auch keine sstädtischen< Probleme mehr, sondern snur noch gesellschaftliche Probleme, die in den Städten manifeste Gestalt annehmen ${ }^{45}$ Zwar sei die Stadt auch heute noch ein besonderer Ort, doch fungiere sie nicht mehr als "Generator ", sondern lediglich als "Katalysator, Filter oder Kompressor gesellschaftlicher Entwicklungen ${ }^{46}$ Die sozialräumliche Struktur

38 Ebd., S. 498.

39 Häußermann, Siebel 2004, S. 99.

40 Häußermann, Siebel 1978, S. 499.

41 Ebd., S. 496.

42 Vgl. ebd., S. 486; Siebel 2012.

43 Häußermann, Siebel 1978, S. 497.

44 Häußermann, Siebel 2004, S. 90.

45 Ebd., S. $91 \mathrm{f}$.

46 Ebd., S. 100. 
der Stadt, die im späten 20. Jahrhundert durch sich verschärfende Segregationsprozesse gekennzeichnet sei, könne zwar "selbstverstärkende Effekte" haben, indem diese »soziale Ungleichheiten vertiefen und so zur Verschärfung sozialer Konflikte beitragen $«{ }^{47}$ In diesen Effekten sehen Häußermann und Siebel jedoch lediglich »Kontexteffekte «. ${ }^{48}$

Zusammenfassend lässt sich festhalten, dass »Stadt « in den Publikationen Häußermanns und Siebels in den vergangenen drei Jahrzehnten » als Ausdruck übergeordneter, allgemeiner gesellschaftlicher Strukturen und Entwicklungen ${ }^{49}$ konzeptualisiert wurde. Ähnlich wie "Stadt « ist auch »Raum « nur Erscheinungsform gesellschaftlicher Strukturen, aber kein eigenständiger soziologischer Gegenstandsbereich. ${ }^{50}$ Einen solchen konstituiert allein » Gesellschaft «, worunter wiederum eine sehr spezifische Gesellschaft, nämlich die entwickelte spätkapitalistische, nationalstaatlich verfasste und westeuropäische (Industrie-)Gesellschaft verstanden wird. "Stadtsoziologie « ist folglich eine Teildisziplin der deutschen Soziologie, die das Klassenverhältnis - konkret: soziale Ungleichheiten in Städten - untersuchen und dabei den Zusammenhang zwischen Stadt- und Gesellschaftsentwicklung thematisieren soll - und die insofern kritisch ist, als dass ihr Anspruch lautet, herrschende Diskurse über Stadt zu identifizieren und öffentlich zu hinterfragen sowie den Blick auf soziale Ungleichheiten in der Stadt zu richten und ihre gesellschaftlichen Ursachen aufzudecken.

\subsection{Die Eigenlogik der Städte}

Ein gänzlich anderer Zugang zur Stadt und damit eine andere Auffassung von Stadtsoziologie wurde unter der Bezeichnung »Eigenlogik der Städte « in den letzten Jahren vor allem am Forschungsschwerpunkt "Stadtforschung " der Technischen Universität Darmstadt entwickelt. Dieser Ansatz wurde erstmals im Jahr 2005 in der Einleitung des Sonderbands der Zeitschrift Soziale Welt mit dem Titel »Die Wirklichkeit der Städte " von Helmuth Berking und Martina Löw skizziert ${ }^{51}$ und 2008 im Sammelband »Die Eigenlogik der Städte « 52 ausgearbeitet. Im Folgenden wird im Unterschied zur Darstellung der kritischen Stadtsoziologie keine Rekonstruktion des Eigenlogik-Ansatzes selbst versucht, ${ }^{53}$ sondern es werden die soziologischen Theorietraditionen herausgearbeitet, die dem Ansatz zugrunde liegen. Namentlich handelt es sich um eine wissens- und kultursoziologische (1), eine raumtheoretische (2) und eine praxeologische Perspektive (3), die in ihren Konse-

47 Ebd.

48 Ebd.; zur Stadt als Ursache sozialer Ungleichheit vgl. Siebel 2012.

49 Häußermann, Siebel 2004, S. 90.

50 Häußermann, Siebel 1978.

51 Berking, Löw 2005, S. 12, S. 18.

52 Berking, Löw 2008 a.

53 Zur Vorstellung des Eigenlogik-Ansatzes selbst sei auf den Aufsatz von Helmuth Berking in diesem Heft verwiesen. 
quenzen, verglichen mit dem Ansatz der kritischen Stadtsoziologie, zu einer Verschiebung des Verständnisses von »Stadt ", »Raum « und »Gesellschaft " führen.

Das Konzept der Eigenlogik der Städte entfaltet sich (1) vor einem wissens- und kultursoziologischen Hintergrund, der systematisch darauf verwiesen ist, die Alltagswirklichkeit hinsichtlich ihres sinnhaften Aufbaus ${ }^{54}$ ernst zu nehmen. Aus dieser Perspektive sind Städte, ihre Unterschiedlichkeit und ihre Besonderheiten nicht nur Elemente dieser Alltagswirklichkeit; sie scheinen in den letzten Jahren im Zuge der Verschränkung von Globalisierung und Lokalisierung zusätzlich an Bedeutung gewonnen zu haben. ${ }^{55}$ Entsprechend des Thomas-Theorems, nach dem das, was die Menschen als real definieren, in seinen Konsequenzen real wird, ${ }^{56}$ und mit dem Wissen, dass auch jeder wissenschaftliche Zugriff auf die Welt seinen unhintergehbaren Ausgangspunkt in der Alltagswirklichkeit findet, ${ }^{57}$ muss die Stadt als soziologischer Gegenstand ernst genommen werden. Die VertreterInnen des EigenlogikAnsatzes vermeiden so einen epistemologischen Standpunkt, der gleichsam behaupten würde, man verfüge über eine Perspektive, die es erlaube, "wahre" soziale Entitäten (Gesellschaften) von »illusionären « (Städten) zu trennen. Wenn Menschen, so das Argument der EigenlogikerInnen, Städte beispielsweise als unterschiedlich attraktiv, tolerant oder lebenswert ansehen, werden sie unter anderem auf Basis dieses Wissens handeln, und ihr Handeln hat als Konsequenz, dass sich dieser Status manifestiert: Dadurch, dass einzelne Städte als Hochburgen bestimmter Szenen oder als Standorte bestimmter Industrien gelten, verstärkt und verfestigt sich die Präsenz dieser Szenen oder Industrien.

Die Frage nach solchen Differenzen zwischen einzelnen Städten lässt sich ohne einen Begriff der Stadt nicht adressieren. In die kritische Stadtsoziologie hat bislang weder eine wissenssoziologische Perspektive noch der cultural turn in den Sozialwissenschaften Eingang gefunden. Eine wissens- und kultursoziologisch informierte Stadtsoziologie jedoch kann nicht umhin, die »Stadt als distinktives Wissensobjekt der Sozialwissenschaften ${ }^{58}$ zu begreifen, und ist daher genötigt, einen Begriff von »Stadt « auszuarbeiten.

Im Zuge der Entwicklung eines allgemeinen Stadtbegriffs, die folgerichtig als Rekonstruktion der Sinneinheit »Stadt « ihren Ausgang nimmt, rekurriert der Eigenlogik-Ansatz neben der wissens- und kultursoziologischen zentral noch auf eine weitere (2) Perspektivumstellung, die im Rahmen des cultural turn mitläuft: den spatial turn. Die Stadt ist demnach - jenseits jeder konkreten inhaltlichen Bestimmung - zunächst einmal eine raumstrukturelle Form. Der zugrunde gelegte Raumbegriff geht über ein Verständnis von Raum als gegebenem physisch-materiellen Behälter, in dem sich soziale Prozesse abspielen, hinaus. Vielmehr wird davon ausgegangen, dass Räume (wie beispielsweise Nationalstaaten oder Heimaten) selbst

54 Schütz 1974.

55 Berking 1998; Berking 2002; Berking 2006; Schroer 2006.

56 Thomas 1928.

57 Berger, Luckmann 2004 [1966].

58 Berking, Löw 2008 b, S. 9. 
in sozialen Prozessen und spezifischen historischen Situationen konstituiert werden. Der Raum ist also nicht länger als unabhängige Variable konzipiert, sondern wird selbst zum Gegenstand der Forschung. In der sozialen Wirklichkeit ist eine Vielzahl zumeist institutionalisierter Raumkonstitutionen anzutreffen, die durch die Zusammenfassung spezifischer Elemente (Syntheseleistung) und durch die relationale Anordnung dieser Elemente (»Spacing «) sozial erzeugt werden und selbst wiederum soziale Wirkmacht entfalten, weil sich die Menschen im Alltag mit Hilfe dieser institutionalisierten und daher objektivierten Raumkonstitutionen orientieren. ${ }^{59}$ Institutionalisierung bedeutet in diesem Zusammenhang, dass ein geteiltes Wissen darüber zur Verfügung steht, welche Elemente zu einer spezifischen Raumkonstitution gehören und wie diese Elemente platziert sein müssen. Die permanente Aktualisierung von Raumkonstitutionen vollzieht sich zumeist im praktischen Bewusstsein. ${ }^{60}$ Das heißt, sie ist diskursiv nicht verfügbar, sondern Element einer präreflexiven Alltagspraxis, sodass die Menschen ohne Weiteres erkennen können, in welchen Räumen sie sich befinden (beispielsweise in einer Kirche), und sich über Räume verständigen können, ohne ihre Elemente und deren Anordnung zu benennen.

Entsprechend dieser Überlegungen handelt es sich aus Sicht der EigenlogikerInnen bei Städten um soziale Raumkonstitutionen. In Anlehnung an Gerd Held wird argumentiert, die moderne Großstadt sei mit der Industrialisierung entstanden, als sich die räumlichen Prinzipien differenzierten, mit Hilfe derer Inklusion und Exklusion organisiert wurden. ${ }^{61}$ Die Städte verzichteten demnach auf die Kontrolle ihrer Grenzen und gewährten freien Zugang für alle. Seitdem sorge die Großstadt durch Verdichtung für Inklusion von Heterogenem, wohingegen der territorial organisierte Nationalstaat Exklusion nach außen (durch Grenzkontrollen) und Homogenisierung nach innen (als Raum gleicher Rechte und Normen) erzeuge. Die beiden komplementären räumlichen Organisationsformen »Großstadt " und »Territorium « bildeten so das subtile raumstrukturelle Fundament der Moderne. ${ }^{62}$

Die raumstrukturelle Form der Großstadt lässt die Alltagspraxis ihrer BewohnerInnen nicht unberührt. Somit finden (3) praxeologische Überlegungen im Rahmen soziologischer Theoriebildung Eingang in die Konzeptualisierung der Eigenlogik der Städte. Damit schließt der Eigenlogik-Ansatz, raumtheoretisch gewendet, an die Überlegungen von Georg Simmel ${ }^{63}$ und Louis Wirth ${ }^{64}$ an. Die Großstadt ist der Ort, an dem wir unablässig Fremden und Fremdem begegnen und mit Reizen überflutet werden, sodass er zu einer distanzierten, blasierten und indifferenten Haltung nötigt. Die Großstadt, so schreibt Berking, »verfügt, verordnet, erzwingt und

59 Löw 2001.

60 Giddens 1988.

61 Held 2005.

62 Ebd., S. 365 f.

63 Simmel 1957 [1903].

64 Wirth 1974 [1938].

Leviathan, 41. Jg., 2/2013 
ermöglicht die Habitualisierung des Unsicheren und Fremden « ${ }^{65}$ Durch Verdichtung werde ein Ort in Form gemeinsamer Routinen und Sinnkontexte in einem Wirklichkeit stiftenden Sinn geschlossen, sodass er als Einheit gedacht werden kann. ${ }^{66}$ Die Verdichtungsleistung der Großstadt erzeugt, wie Ulf Matthiesen im Anschluss an die phänomenologische Theorietradition von Schütz argumentiert, ein »lebensweltliches Hintergrundwissen ${ }^{67}$ Bei diesem Wissen handelt es sich zunächst nicht notwendig um reflexiv verfügbares Wissen, sondern es ist in der Routine und Alltagspraxis der StädterInnen aufgehoben. Damit ergibt sich im Rahmen des Eigenlogik-Ansatzes eine zweite Ebene, auf der die Unterschiedlichkeit von Städten thematisiert werden kann. Städte sind nicht nur unterschiedliche Wissensobjekte im Rahmen einer wissenssoziologischen Betrachtung, sondern sie generieren aufgrund ihrer speziellen Verdichtungsleistungen auch unterschiedliche Formen der Alltagspraxis, die sich präreflexiv in die habituellen Dispositionen ihrer BewohnerInnen einschreiben.

Das gemeinsame theoretische Fundament einer Konzeption der »Eigenlogik der Städte " lässt sich also bestimmen als (1) kultur- und wissenssoziologischer Zugriff, der die Stadt aufgrund ihrer (Handlungs-)Relevanz in der Alltagswirklichkeit als sozialwissenschaftlichen Gegenstand ernst nimmt, (2) als raumtheoretisch fundiertes Programm, das die Stadt als eine spezifische raumstrukturelle Form begreift, die durch Verdichtung und den Verzicht auf Grenzkontrollen Inklusion erzeugt und Heterogenität organisiert, und (3) als praxissoziologisch informierter Ansatz, der Städten zutraut, die Alltagspraxis ihrer BewohnerInnen mit zu formen.

Gleichwohl lässt sich die Perspektive »Eigenlogik der Städte « konzeptuell nicht als monolithischer Block beschreiben. Auf dem hier skizzierten Theoriefundament entwickeln sich durchaus unterschiedliche Ansätze zur Erforschung von städtischer Eigenlogik. Während Helmuth Berking eher auf zeichentheoretischer Basis die kognitive Herstellung des sinnhaften Ganzen der Stadt in den Blick nimmt, lenkt Martina Löw den Fokus der Aufmerksamkeit in eine andere Richtung. Vor dem Hintergrund der Giddens'schen Strukturationstheorie, mit der auch Städte als strukturierende Strukturen begriffen werden können, wendet sie sich stärker den spezifischen Alltagspraktiken in Städten zu, die über ihre Körperlichkeit den Habitus der BewohnerInnen formen. ${ }^{68}$ »Das Konzept der Eigenlogik der Städte trägt in sich [...] die Annahme, dass Städte Sinnwelten darstellen, die in den Habitus der Bewohner eingehen ${ }^{69}$ Helmuth Berking hingegen fokussiert stärker auf die historische Entwicklung stadtspezifischer Sinnbezüge, die zu einer je eigenen »kumulativen Textur «, zu einem je eigenen »Bedeutungsgewebe der Stadt « ${ }^{70}$ aufgeschichtet werden. Auf dieser Grundlage entstünden doxische Gewissheiten, worunter Berking ein

65 Berking 2008, S. 26.

66 Frank 2012, S. 269.

67 Matthiesen 1997.

68 Löw 2008 b; Löw 2011.

69 Löw 2011, S. 88 f.

70 Berking, Schwenk 2011, S. 21. 
ortsspezifisches, quasi natürliches In-der-Welt-Sein versteht: »Stadt< geht mit Wahrnehmungs- und Gefühls-, mit Handlungs- und Deutungsschemata einher, die in ihrer Gesamtheit das ausmachen, was man als 'großstädtische Doxa bezeichnen kann ${ }^{7}{ }^{71}$

\subsection{Fazit: Differierende Grundpositionen}

Aus der obigen Darstellung der theoretischen Fundierungen der kritischen Stadtsoziologie einerseits und des Ansatzes der Eigenlogik der Städte andererseits lassen sich einige fundamentale Diskrepanzen herauslesen. Die unterschiedlichen Auffassungen von Stadtsoziologie, die ihren Kern in den unterschiedlichen Theorietraditionen haben, lassen sich am besten veranschaulichen, wenn wechselseitig die Einwände gegen die jeweils andere Perspektive expliziert werden. Zunächst werden zwei Einwände der kritischen Stadtsoziologie gegen den Ansatz der Eigenlogik der Städte dargestellt, um anschließend aus eigenlogischer Perspektive zwei Kritikpunkte an der kritischen Stadtsoziologie zu formulieren. ${ }^{72}$

Der erste Einwand der kritischen Stadtsoziologie fragt nach dem kritischen Potenzial des Eigenlogik-Ansatzes. Der Anschluss an die lebensweltlichen Sinnhorizonte, der im Rahmen des Eigenlogik-Konzepts mitgeführt wird, provoziere demnach die Verschleierung von klassenspezifischen Interessenlagen, deren Ursache eben nicht auf Stadtebene gesucht werden könne, sondern auf Ebene der Gesellschaft. ${ }^{73}$ Daher drohe bei der eigenlogischen Stadtforschung eine affirmative Haltung unter anderem gegenüber den an wirtschaftlichen Interessen orientierten, professionell produzierten Selbstdarstellungen von Städten und gegenüber lokalpatriotischen Stimmungslagen (vgl. hierzu ausführlicher Kapitel 3).

Zudem spielt der Raum in der kritischen Stadtsoziologie eine der Gesellschaft untergeordnete Rolle. Er erscheint einzig als eine Folie, auf der sich Klassenkonflikte in Form von Segregationseffekten ablesen lassen. In keinem Fall kann er selbst als unabhängige Variable ins Feld geführt werden, wie der Eigenlogik-Ansatz mit seinem Fokus auf raumstrukturelle Form und daraus abgeleiteten Verdichtungs- und Heterogenisierungsprozessen behauptet. Aus Sicht der kritischen Stadtsoziologie wird hier Soziales mit Nicht-Sozialem erklärt und damit das Feld legitimer soziologischer Forschung verlassen.

Aus Sicht des Eigenlogik-Ansatzes wird zunächst konstatiert, dass der kritische Ansatz Stadtforschung ohne Stadt ${ }^{74}$ betreibe. »Die stadtsoziologische Forschung ohne Stadt ist nicht nur blind für die Differenz zwischen Städten, für die Eigenlogiken und lokalen Kontextbedingungen >dieser im Unterschied zu >jener Stadt, sondern auch für die `Stadt $<$ als Wissensobjekt selbst $« .{ }^{75}$ Dies wird vor allem mit Blick auf die Selbstverständlichkeit diskutiert, mit der die (nationalstaatliche)

71 Berking 2008, S. 23.

72 Vgl. ausführlich die Beiträge von Berking und Siebel in diesem Heft.

73 Vgl. zum Beispiel Michel 2011.

74 Berking, Löw 2008 a.

75 Berking 2008, S. 16.

Leviathan, 41. Jg., 2/2013 
Gesellschaft von der kritischen Stadtsoziologie als Generator alles Sozialen konzipiert wird. Was, so fragen die EigenlogikerInnen, macht die VertreterInnen der kritischen Stadtsoziologie eigentlich so sicher, dass Vergesellschaftung einzig auf Ebene des Nationalstaates stattfindet und nicht (auch) auf Ebene der Stadt?

Dieser Einwand gegenüber dem kritischen Ansatz wird ergänzt durch den Verweis, dass die Spezifik einzelner Städte und die Differenzen zwischen ihnen in den Alltagsgewissheiten der Menschen einen festen Platz hätten und somit handlungswirksam würden. Eine Erklärung für dieses aus wissenssoziologischer Perspektive sozial relevante Phänomen hat die kritische Stadtsoziologie nicht anzubieten. Für die EigenlogikerInnen ist nicht nachvollziehbar, dass Gesellschaften »realer» als Städte seien.

Festhalten lässt sich, dass sich hinter dem Perspektivenstreit in der Stadtsoziologie die Differenz zwischen einer historisch-materialistischen Auffassung von (Stadt-)Soziologie und einer kultur- und wissenssoziologisch fundierten, raum- und praxistheoretisch informierten Perspektive verbirgt. Damit verweist die hier umrissene Auseinandersetzung zugleich auch auf eine aktuelle Debatte um den Gegenstandsbereich und die Aufgaben der Soziologie als Ganzer.

\section{Die Kontroverse um die These der Eigenlogik}

Das Konzept der städtischen Eigenlogik hat eine Reihe von Diskussionen ausgelöst, die im Folgenden dargestellt werden. Wir beginnen mit den kritischen Einwänden gegen die Eigenlogik-Perspektive; es folgen produktive Aufnahmen dieses Forschungsansatzes vor allem in den Nachbardisziplinen.

\subsection{Kritik}

Die Kritik am eigenlogischen Forschungsprogramm umfasst maßgeblich fünf zentrale Rückfragen oder Einwände, die erstens den Status des Ansatzes der »Eigenlogik der Städte" selbst betreffen, zweitens die geringe empirische Basis des Forschungsansatzes bemängeln, drittens die Verwendung der Begriffe »Habitus « und »Doxa « problematisieren, viertens eine Kulturalisierung, Subjektivierung und Substantialisierung von »Stadt " befürchten und schließlich fünftens eine affirmative Haltung bei der Suche nach städtischen Eigenlogiken am Werke sehen.

\section{Status der »Eigenlogik»}

Aus vielen kritischen Reaktionen auf die Perspektive der Eigenlogik der Städte spricht eine Unsicherheit darüber, in welcher Weise dieses neue Forschungsprogramm auf die etablierte, kritische Stadtsoziologie Bezug zu nehmen beabsichtigt. Dabei schwanken die Einschätzungen zwischen den Polen der »Ablösung « und der »Ergänzung « der etablierten Forschungsrichtung. Tatsächlich sprechen Berking und 
Löw einerseits von einem »Perspektivwechsel « ${ }^{76}$, andererseits artikulieren sie, »systematische Blindstellen « überwinden und »[n]icht länger und ausschließlich in den Städten forschen, sondern die Städte selbst erforschen " $77 \mathrm{zu}$ wollen und somit nach nicht weniger als »nach einer Theorie der Stadt « zu suchen. ${ }^{78}$ Entsprechend fragt Hartmut Häußermann, ob es sich beim eigenlogischen Forschungsansatz um einen Paradigmenwechsel handele oder »(lediglich) um eine stärkere Betonung lokal spezifischer Traditionen, Kulturen, Mentalitäten oder Praxen, deren Existenz niemand bestreiten kann «. ${ }^{79}$ Auch Jens Wietschorke ${ }^{80}$ scheint sich in der Einschätzung des eigenlogischen Forschungsprogramms vor dieselbe Frage gestellt zu sehen. Seine Antwort lautet, dass die Thesen zur »Eigenlogik der Städte« zwar nicht »zur Etablierung einer alternativen Stadtsoziologie « 81 taugen, sie aber »durchaus eine erhellende Ergänzung zur strukturellen und sozialräumlich argumentierenden Stadtsoziologie bilden $«{ }^{82}$ Auch in anderen Stellungnahmen geht der Tenor in diese Richtung. Gabriela B. Christmann ${ }^{83}$ sieht die Erforschung städtischer Eigenlogiken als innovativen Diskussionsstrang in der Stadtsoziologie und als neuen Forschungszweig, dessen Etablierung sie für unsicher, aber für »dringend notwendig « 84 hält. Und auch Boris Michel ${ }^{85}$ räumt ein, dass es »eine Reihe berechtigter Kritiken an der 'Neuen Stadtsoziologie und dieser nahestehenden Ansätzen in der Stadtforschung gebe und der eigenlogische Ansatz wichtige Fragen aufwerfe «. ${ }^{86}$ Diese Reaktionen zeigen, dass die dem Forschungsprogramm einer Eigenlogik der Städte zugrunde liegenden Anstöße wie auch die darüber artikulierten Fragen - auch an die kritische Stadtsoziologie - durchaus als berechtigt betrachtet werden, zumindest solange sich der Eigenlogik-Ansatz als Ergänzung oder Bereicherung der bisherigen Forschungstradition begreifen lässt, das bisherige Paradigma also nicht grundlegend infrage gestellt werden soll. Starken Dissens und massive Bedenken gibt es allerdings auf der Ebene der konzeptuellen Umsetzung der den Ansatz der Eigenlogik der Städte anleitenden Fragen.

76 Berking, Löw 2008 b, S. 7.

77 Ebd.

78 Berking 2008, S. 18.

79 Häußermann 2011, S. 163.

80 Wietschorke 2012.

81 Ebd.

82 Ebd.

83 Christmann 2011.

84 Ebd., S. 398.

85 Michel 2011.

86 Ebd., S. 119. 


\section{Probleme der Empirie}

An verschiedenen Stellen richtet sich die Kritik auf die empirischen Grundlagen der eigenlogischen Stadtforschung. Boris Michel moniert in seiner Besprechung des Sammelbands »Die Eigenlogik der Städte « ${ }^{87}$, es handle sich bei der Eigenlogik der Städte um ein "weitgehend abstraktes 'Nachdenken über die Stadt ohne Bezugnahme auf die empirische Stadtforschung « ${ }^{88}$ Bemängelt wird hier schlicht das Fehlen empirischer Studien, die dem eigenlogischen Hypothesenrahmen die nötige Plausibilität verleihen könnten. ${ }^{89}$

An den von Martina Löw in ihrer Monografie »Soziologie der Städte « ${ }^{90}$ präsentierten Studien zu Berlin und München, ${ }^{91}$ denen sie allerdings explizit einen »vorbereitenden Charakter " zuschreibt, kritisiert Jens Kastner ${ }^{92}$ ebenfalls ein Zurückfallen der Empirie hinter die Theorie, ${ }^{93}$ was sich insbesondere in der Reduktion von Stadtbildern auf im Rahmen von Werbekampagnen produzierte Repräsentationsformen äußert, wodurch »jene verkörperten Praxen aus dem analytischen Rahmen, die im theoretischen Teil noch als wichtig galten " ${ }^{94}$ verbannt werden. Jens Wietschorke ${ }^{95}$ weist in seiner Diskussion desselben Bands auf das Fehlen der sozial- und kunsthistorischen Tiefendimension hin, wodurch die empirischen Analysen an der Oberfläche bleiben. Insgesamt unterläuft seinem Eindruck nach die dort präsentierte Empirie die Unterscheidung zwischen der professionellen Inszenierung des Eigenen der Stadt und der gesuchten Eigenlogik.

\section{Die Begriffe »Habitus « und »Doxa«}

Ebenfalls Anlass zur Kritik liefert die Verwendung der Begriffe »Habitus «96 und »Doxa « ${ }^{97}$ in verschiedenen Publikationen zur Eigenlogik der Städte. Insbesondere

87 Berking, Löw 2008 a.

88 Michel 2009, S. 353. Eine ähnliche Kritik trägt auch Ariane Sept (2009) vor.

89 Helmuth Berking und Martina Löw haben dieses Defizit selbst thematisiert: »Der Stadtforschung fehlt das empirische Wissen über die eigenlogische Entwicklung von Städten und deren Rahmenbedingungen « (Berking, Löw 2008 b, S. 12). Mittlerweile liegen allerdings erste, abgeschlossene Studien vor, die sich einer eigenlogischen Frageperspektive verpflichtet fühlen (vgl. beispielsweise Berking, Schwenk 2011; Berking, Frank 2010).

90 Löw 2008 b.

91 Vgl. ebd., S. 187-230.

92 Kastner 2009 a; Kastner 2009 b.

93 Vgl. Kastner 2009 a, S. 352.

94 Vgl. ebd.

95 Wietschorke 2009.

96 Vgl. beispielsweise Löw 2008 a, S. 40-45; Bockrath 2008; Lindner 2008, S. 87 ff.

97 Berking 2008, S. 24-28; Löw 2008 a, S. 40-45. 
um das Habitus-Konzept wird auch in den Reihen der EigenlogikerInnen eine Auseinandersetzung geführt. ${ }^{98}$

Rolf Lindner ${ }^{99}$ hat im Anschluss an Martyn Lee ${ }^{100}$ das Habitus-Konzept genutzt, um eine » Theorie städtischer Individualitäten « $101 \mathrm{zu}$ entwickeln. Städte bilden, so die These, in Abhängigkeit zu ihren jeweils zentralen ökonomischen Sektoren spezifische Geschmackslandschaften aus, die sich derart verfestigen, dass - vergleichbar mit einer Biografie - »bestimmte Entwicklungslinien näher liegen, andere ferner stehen « ${ }_{0}^{102}$ Diese symbolischen Verfestigungen fasst Lindner als »Habitus der Stadt «103, um so die Unterschiede zwischen Städten konzeptuell zugänglich zu machen.

Martina Löw ${ }^{104}$ und Franz Bockrath ${ }^{105}$ stehen diesem Unternehmen allerdings kritisch gegenüber. Problematisch erscheint die implizite Gleichsetzung von Körper und Stadt, ${ }^{106}$ was einerseits das Risiko eines » vereinfachende[n] Anthropomorphismus « 107 in sich trage und andererseits auch das systematische Problem aufwerfe, »dass der Habitus von soziologischen Strukturkategorien - wie etwa Geschlecht, Klasse, Feld - nicht zu trennen ist «. ${ }^{108}$ Für Löw liegt genau darin das Problem eines »Habitus der Städte«. Wird der Habitus einer Stadt in Analogie zum Habitus eines Menschen konzeptualisiert, müssten sich außerhalb der Stadt liegende Faktoren angeben lassen, die zur Prägung eines solchen Habitus beitragen. Der Versuch Lindners, den »Habitus der Städte « aus der internen Ökonomie einer Stadt heraus zu erklären, vermag Löw daher nicht zu überzeugen. Sie kritisiert, dass, wenn man »die Eigenlogik der Stadt mit dem Habitusbegriff « erkläre, unklar bleibe, »was also jene konditionierenden Relationen sein könnten, welche den Habitus der Stadt weitgehend determinieren ${ }^{1}{ }^{109}$

Die kritische Auseinandersetzung mit dem »Habitus der Stadt « führt bei Löw und Bockrath allerdings nicht zur Verabschiedung des Habitus-Konzepts, sondern zu dessen praxeologisch gewendeter Wiederaufnahme als »städtischer Habitus «. 110 »Das Konzept der Eigenlogik der Städte«, schreibt Löw, »trägt in sich umgekehrt

98 Vgl. dazu Bockrath 2008; Löw 2008 b, S. 87-91; zusammenfassend: Frank 2012, S. 292-293.

99 Lindner 2008.

100 Lee 1997.

101 Lindner 2008, S. 84.

102 Ebd., S. 87.

103 Lindner 2003.

104 Löw 2008 b, S. 87-91.

105 Bockrath 2008.

106 Vgl. Löw 2008 b, S. 87.

107 Bockrath 2008, S. 71.

108 Ebd.

109 Löw 2008 b, S. 88.

110 Bockrath 2008, S. 62. 
die Annahme, dass Städte Sinnwelten darstellen, die in den Habitus der Bewohner eingehen " ${ }^{111}$ Der »städtische Habitus " setzt gewissermaßen eine Ebene tiefer an und versucht den »wechselseitigen Zusammenhang zwischen übergreifenden städtischen Strukturen und hierauf bezogenen Praktiken städtischer Akteure «112 aufzuklären. Er zielt also auf die strukturierenden und strukturierten sozialen Praktiken der Akteure in der Stadt.

Forschungslogisch hat der von Helmuth Berking zur Diskussion um die "Eigenlogik der Städte " beigetragene Begriff der »Doxa « auf dieser Ebene seinen Einsatzpunkt. ${ }^{113}$ Der (sozial)phänomenologischen Tradition verpflichtet, verweist Doxa für Berking zunächst auf eine » auf Fraglosigkeit und Vertrautheit basierende >natürliche< Einstellung zur Welt «. ${ }^{114}$ Allerdings erfährt dieses Konzept bei Berking eine Erweiterung. Dabei betont er insbesondere, dass ebenso die Erfahrung von Räumen und Orten doxisch sein kann, wodurch auch die Stadt als »lebensweltliches Hintergrundwissen « 115 Berücksichtigung erfahren muss. Damit rückt - im Unterschied zum Konzept eines »städtischen Habitus « - das implizite, aber handlungsleitende Wissen, das Städte den Menschen »aufdrängen «, in den Fokus des Interesses.

Aus einer dem Eigenlogik-Ansatz kritisch gegenüberstehenden Perspektive hat Norbert Gestring ${ }^{116}$ einige grundsätzliche Einwände gegen die Verwendung von Konzepten wie Habitus und Doxa in der eigenlogischen Stadtforschung vorgetragen. Problematisch erscheint ihm vor allem der Gedanke eines "städtischen Habitus «, weil dadurch der Stadt eine höhere Prägekraft zugesprochen würde als sozialen Schichten und Milieus. ${ }^{117}$ Generell bemängelt er: »Die Versuche, einen städtischen Habitus oder einen Habitus der Stadt zu finden, unterstellen eine Homogenität, die es angesichts der ökonomischen, sozialen und kulturellen Vielfalt (nicht nur) in deutschen oder europäischen Städten nicht geben kann «. ${ }^{118}$ Der Verwendung des Doxa-Konzepts, wie es Helmuth Berking für die Eigenlogik der Städte vorgeschlagen hat, attestiert Gestring - im Gegensatz zu der bei Bourdieu ${ }^{119}$ mit diesem Begriff verbundenen Intention - den Verlust des kritischen Potenzials. Zweifelhaft erscheint ihm auch, ob es überhaupt so etwas wie eine städtische Doxa geben kann. So kommt Gestring schließlich zu der Einschätzung, dass sich mit Bourdieus Konzepten von Habitus und Doxa »der Ansatz der >Eigenlogik der Städte`nicht begründen «120

111 Löw 2008 b, S. 89.

112 Bockrath 2008, S. 62.

113 Vgl. Berking 2008, S. 24-28.

114 Ebd., S. 24; vgl. Matthiesen 1997.

115 Berking 2005, S. 25.

116 Gestring 2011.

117 Vgl. ebd., S. 44.

118 Ebd., S. 46.

119 Ebd., S. 48.

120 Ebd., S. 51. 
lässt. Ähnlich argumentiert auch Stefan Höhne ${ }^{121}$, der bei der Verwendung des Habitus- und Doxa-Konzepts im Rahmen des eigenlogischen Forschungsprogramms eine "Abblendung von Prozessen sozialer Ungleichheit zugunsten einer Betonung der homogenen Doxa als im höchsten Maß inkludierende und nivellierende Instanz «122 am Werke sieht. Die Ungleichheitskategorien Geschlecht, Klasse und Bildung erführen so eine systematische Abwertung bei gleichzeitiger Aufwertung »der Wirkmächtigkeit des lokalen Habitus der ganzen Stadt «. ${ }^{123}$

\section{Die Gefahr von Kulturalisierung, Subjektivierung, Substantialisierung}

Eng verbunden mit der Kritik am Habitus- und am Doxa-Konzept der eigenlogischen Forschungsperspektive ist der beispielsweise von Jan Kemper und Anne Vogelpohl vorgetragene Einwand, der eigenlogische Forschungsansatz betreibe eine »Kulturalisierung der Städte «. ${ }^{124}$ Unter Kulturalisierung verstehen die AutorInnen allgemein Argumentationen, die Kultur »zur zentralen erklärenden Variable für Prozesse sozialen Handelns und Verhaltens, letztlich für soziale Ordnungsbildung « 125 erheben. Bei der eigenlogischen Forschungsperspektive sehen sie eine solche Argumentation am Werk, insofern dort den »verborgenen Strukturen [...], die sich stillschweigend und mit Regelmäßigkeit zur Geltung bringen ${ }^{126}$ und die im Wesentlichen als » kulturelle Strukturen « 127 verstanden würden, besondere Bedeutung zukäme. Dadurch werde ein »Erklärungsmodell formuliert, das ideelle bzw. kognitive Strukturen zum determinierenden Moment gesellschaftlicher Entwicklung macht «. ${ }^{128}$ Die Städte würden so mit einer »kulturellen Autonomie «129 ausgestattet, die eine »Homogenisierung von Erfahrungswelten und deren Entkopplung von politischen, ökonomischen und sozialen Kontexten« zur Folge hätte.

In eine ähnliche Richtung zielen Kritiken, der eigenlogische Forschungsansatz betreibe eine Subjektivierung und Substantialisierung von Stadt. Auf der Suche nach den Differenzen zwischen den Städten und damit auf der Suche nach deren Eigenarten - so das Argument - werde, um entsprechende Attribuierungen vornehmen zu können, die Stadt in der eigenlogischen Begriffsbildung als Subjekt gedacht. ${ }^{130}$ Damit verbunden sei, wie schon bei der kritischen Bewertung des Habitus-Konzepts und dem Vorwurf einer »Kulturalisierung der Städte" moniert, eine begriffliche

\section{Höhne 2011.}

122 Ebd., S. 64.

123 Ebd.

124 Kemper, Vogelpohl 2011, S. 31.

125 Ebd.

126 Ebd., S. 19; Hervorhebung im Original.

127 Ebd., S. 20; Hervorhebung im Original.

128 Ebd., S. 31.

129 Ebd., S. 26.

130 Vgl. Michel 2011, S. 131. 
Homogenisierung städtischer Wirklichkeit auf Kosten der verschiedenen Ungleichheitskategorien: Wenn die VetreterInnen des eigenlogischen Forschungsansatzes »die Stadt als individuelle, und damit unteilbare, Ganzheit erforschen wollen, reproduzieren sie eine Vereindeutigungs- und Subsumtionslogik, die Konflikte und differente Interessen unterbelichtet «. ${ }^{131}$ Darüber hinaus werde einer Substantialisierung von Städten das Wort geredet: »Dieser Komplex von Einstellungen, Ideologien, Selbstbildern und Mentalitäten, der von Stadt zu Stadt unterschiedlich sein kann ", schreibt Hartmut Häußermann, wird »als >Eigenlogik « bezeichnet und zum zentralen Forschungsgegenstand erklärt. Lokale kulturelle Variationen werden damit substantialisiert. Städte gewinnen sogar eine eigene Persönlichkeit «. ${ }^{132}$ Kritisiert wird also, dass den im eigenlogischen Denken konstruierten "Stadtsubjekten " wesenhafte Zuschreibungen zugeordnet würden, die dann jenseits aller historisch beobachtbaren Brüche und Wandlungen Gültigkeit besitzen sollen.

\section{Affirmative Eigenlogik}

Die Warnungen vor einem Hang zum Subjektivismus und Substantialismus führen schließlich zu einer letzten Kategorie von kritischen Einwänden gegen das Konzept der Eigenlogik der Städte. Angemahnt wird, dass sich die den »Stadtsubjekten « als Differenzmarkierungen zugeschriebenen Eigenschaften in einer allzu großen Nähe $\mathrm{zu}$ entsprechenden Imagekampagnen der städtischen Werbeagenturen bewegen würden und der Eigenlogik-Ansatz damit affirmativ wirke und als Stichwortgeber für die Vermarktung von Städten fungiere. Der dahinter liegende Vorwurf zielt auf die Gegenstandsebene des eigenlogischen Unternehmens, insofern damit der Verdacht ausgesprochen wird, es gäbe eine problematische und unreflektierte Nähe zwischen städtischen Werbeagenturen und Wissenschaft: "Damit eignet sich der 'Eigenlogik«-Ansatz bestens für städtische Imagekampagnen und die Inszenierung lokaler Differenz im Wettbewerb der Städte «. ${ }^{133}$

Vor diesem Hintergrund wird der Perspektive der Eigenlogik der Städte dann eine Tendenz zur Entpolitisierung attestiert, ${ }^{134}$ was Hartmut Häußermann zu der Nachfrage an die Adresse des eigenlogischen Forschungsprogramms veranlasste: »Soll nun endlich die Stadtsoziologie als Krisenwissenschaft überwunden und an ihre Stelle eine - theoretisch nun ganz unvoreingenommene - Beschreibung lokaler Kulturen treten? «135

131 Höhne 2011, S. 66.

132 Häußermann 2011, S. 160.

133 Michel 2011, S. 132; vgl. Wietschorke 2009.

134 Vgl. Bürk 2011, S. 152.

135 Häußermann 2011, S. 164. 


\subsection{Produktive Anschlüsse}

Jenseits der beschriebenen, vorwiegend innerhalb der (kritischen) Stadtsoziologie und der kritischen Geographie geführten Debatten um konzeptuelle Schwächen des Eigenlogik-Ansatzes scheint die Vorstellung, dass Städte spezifische Erfahrungsund Handlungsräume sind, durchaus anschlussfähig an Forschungsfragen einiger Nachbardisziplinen zu sein. Das gilt besonders für die lokale Politikforschung. ${ }^{36}$

Die lokale Politikforschung beschäftigt sich mit Formen des städtischen Regierens und ist seit gut zwei Jahrzehnten auf der Suche nach Erklärungsmöglichkeiten für »die offenkundigen Differenzen der Städte in der Problemlösung in ihrer sachlichen und politischen Dimension. "137 Gefragt wird etwa, warum ein Phänomen in der einen Stadt als Problem wahrgenommen und bearbeitet wird, während es in einer anderen Stadt keine diskursive oder handlungspraktische Relevanz entwickelt, oder wie es dazu kommt, dass überregional erlassene politische Maßnahmenbündel vor Ort zum Teil sehr unterschiedlich implementiert werden. ${ }^{138}$ Um dies zu erklären, haben Bernhard Blanke, Susanne Benzler und Hubert Heinelt in den 1980er Jahren in ihren Untersuchungen zur lokalen Arbeitsmarktpolitik das Konzept der "Arenafärbung " entwickelt. ${ }^{139}$ Das Konzept berücksichtigt die Verflechtung von politikspezifischen Aspekten mit internationalen, nationalen und regionalen Rahmenbedingungen städtischen Regierens, weist aber - über die Metapher der Färbung - dem stadtspezifischen Kontext eine signifikante Bedeutung zu. Konzeptuell tritt die Vor-Ort-Wahrnehmung von Gewinnen und Verlusten durch lokale Politik in den Vordergrund der Analyse. Formuliert wird somit ein »im Kern interpretatives Vorgehen [...], das lokale Problemkonstruktionen in einen Kontext widerstreitender Interpretationen über mögliche Folgen von Entscheidungen stellt. «140 Diese Interpretationen basieren, wie Karsten Zimmermann hervorhebt, auf unterschiedlichen lokalen Wissensbeständen. ${ }^{141}$ Das Eigenlogik-Konzept, das Städte als »Ensemble zusammenhängender Wissensbestände und Ausdrucksformen «142 versteht und städtisches Handeln praxeologisch (und damit über das für die politische Analyse bestimmende rational choice-Modell hinausgehend) wendet, ist deshalb für die lokale Politikforschung von großem Interesse. Aufschlussreich sei, so Zimmermann, "vor allem die Veränderung der Heuristik im Blick auf die Stadt, [...] da dies mit Veränderungen der handlungstheoretischen Grundannahmen einhergeht. «143

Eine argumentative Nähe zum soziologischen Konzept städtischer Eigenlogik lässt sich auch in der hierzulande vor allem von Rolf Lindner konzeptuell vorangetrie-

136 Vgl. Heinelt, Mayer 2001.

137 Zimmermann 2008.

138 Ebd., S. 213.

139 Blanke et al. 1989.

140 Zimmermann 2012, S. 286.

141 Ebd.

142 Löw 2008 b, S. 78.

143 Zimmermann 2012, S. 287.

Leviathan, 41. Jg., 2/2013 
benen kulturwissenschaftlich-ethnographischen Stadtforschung erkennen. ${ }^{144}$ So steht es für Lindner "außer Zweifel «, dass "Städte individuelle Gebilde sind, mit einer eigenen Biografie (sprich: Geschichte), einer eigenen Sinnesart (state of mind) und ihr eigenen Mustern der Lebensführung «. ${ }^{145}$ Theoretisch bezieht sich Lindner - ähnlich wie Helmuth Berking - auf den symbolisch-repräsentativen Ansatz der Stadtforschung, der auf Arbeiten des Historikers Richard Wohl und des Soziologen Anselm Strauss in den 1950er und 1960er Jahren zurückgeht. ${ }^{146} \mathrm{Im}$ Zentrum der Analyse stehen materielle wie immaterielle Repräsentationen von Städten, also Wahrzeichen, Denkmale und Architekturen ebenso wie Anekdoten, Mythen und Lieder. Diese bilden, so Lindner in Anlehnung an den Stadtsoziologen Gerald D. Suttles ${ }^{147}$, eine »kumulative Textur «, ein durch Aufschichtung und Überlagerung symbolischer Repräsentationen entstehendes »Webmuster «. Der Begriff der »kumulativen Textur ", der auch Eingang in das Eigenlogik-Konzept gefunden hat, ${ }^{148}$ schließt die potentiell konflikthafte Auseinandersetzung um die Deutungshoheit über die Geschichte und Repräsentation einer Stadt nicht aus. In den Blick geraten jedoch vor allem Bezugnahmen auf Althergebrachtes, Selbst- und Fremdtypisierungen, Metonymien und Metaphern. Wie dies konkret im Feld vonstatten geht, haben Rolf Lindner und Johannes Moser empirisch am Beispiel der »symbolischen Residenzstadt « Dresden ${ }^{149}$ und Lutz Musner am Beispiel Wiens ${ }^{150}$ gezeigt. Musner analysiert, ausgehend vom berühmten Canaletto-Blick vom Oberen Belvedere, ${ }^{151}$ die Literatur, Musik und Essenskultur Wiens und beschreibt die Wien-spezifische Codierung der Stadtlandschaft, die widersprüchliche Einheit von Tradition und Moderne in der Architektur sowie die sprichwörtlich gewordene »Wiener Gemütlichkeit «. Er kommt zu dem Schluss, dass im Falle Wiens Traditionen und ästhetische Codierungen die Herausbildung eines ungewöhnlich resistenten Selbstund Fremdbildes befördert haben, das heute wichtiger Bezugspunkt für politisches und ökonomisches Handeln ist.

Ähnlich anschlussfähig scheint das Eigenlogik-Konzept für die Stadtgeschichtsforschung zu sein. Insbesondere die Denkfigur der »kumulativen Textur « öffne, so schreibt Dieter Schott, die sozialwissenschaftliche Stadtforschung für historische Dimensionen. ${ }^{152} \mathrm{Er}$ sieht darin sogar eine Aufwertung der Stadtgeschichte für die gegenwartsbezogene Stadtforschung sowie »eine Basis für interdisziplinäre Forschung « und » für die Integration von Stadtgeschichte in ein breiteres Feld der >urban

144 Vgl. Lindner 2005 b; Lindner 2005 a; Lindner, Moser 2006; Lindner 2008.

145 Lindner 2008, S. 83.

146 Vgl. Wohl, Strauss 1958; Strauss 1961.

147 Vgl. Suttles 1984.

148 Vgl. Berking, Schwenk 2011.

149 Lindner, Moser 2006.

150 Musner 2009.

151 Vgl. ebd., S. 59 ff.

152 Schott 2012. 
studies« «. ${ }^{153}$ Das habe vor allem mit der »hohe[n] Plausibilität« zu tun, die die Grundannahme einer historischen Prägung auch aktueller kultureller Muster für Stadthistoriker habe. ${ }^{154}$ Ferner sieht er erhebliche Überschneidungen zwischen dem Konzept der Eigenlogik und dem kulturalistisch ausgerichteten Zweig der Stadtgeschichte, in welchem in den vergangenen Jahren zahlreiche Arbeiten erschienen, die Diskurse um Stadtbilder und Stadtrepräsentationen untersuchten. ${ }^{155}$ Löblich und für die Stadtgeschichte anschlussfähig findet Schott nicht zuletzt den mit dem Eigenlogik-Konzept verknüpften Versuch, die Materialität der Stadt in der sozialwissenschaftlichen Stadtforschung stärker zu berücksichtigen.

Explizit mit immateriellen Aspekten von Kultur sind die Literatur- und die Medienwissenschaften befasst, und auch hier lassen sich produktive Bezugnahmen auf den Eigenlogik-Ansatz finden. Im Fokus steht bislang die Kriminalliteratur-Forschung, da sich seriell erzählte Geschichten von Tod und Verbrechen offensichtlich »besonders glaubwürdig in der heterogenen und verdichteten Vergesellschaftungsform urbanen Lebens ansiedeln lassen «. ${ }^{156}$ So untersucht ein Sammelband zum deutschen Fernsehklassiker Tatort die Raumbezüge, die diese Krimireihe strukturieren, »von der räumlichen Inszenierung der jeweiligen Stadt in einer einzelnen Folge bis zum kartierenden Netzwerk von Städteverbindungen im Gesamtzusammenhang der Reihe «. ${ }^{157}$ Konzeptuell schlagen die HerausgeberInnen Julika Griem und Sebastian Scholz Funken aus dem - im Fall des Phänomens Tatort geradezu idealtypisch gegebenen - Spannungsfeld zwischen Allgemeinheit (der Tatort als allsonntäglich ähnlich wiederkehrende Krimi-Reihe) und Besonderung (der Tatort als Kriminalgeschichte in einer spezifischen Stadt). Wenn, so fragen sie, mit dem Eigenlogik-Konzept davon ausgegangen werden kann, dass »Städte einen konstitutiven Wahrnehmungs-, Sinn- und Handlungshorizont « 158 darstellen, dann muss ein Vergleich zwischen den Tatort-Folgen unterschiedlicher Städte Aufschluss darüber geben, ob beispielsweise »Täterprofile und Spurensuchen, Ermittlungsstile und Verbrechertypen, Beziehungsformen und Problemlösungsstrategien nur an bestimmten Orten möglich und plausibel oder auch austauschbar sind «. ${ }^{159}$ Konkreter fragen sie: »Ist es aus der Praxis des jeweiligen städtischen Lebens zu begründen oder hat es sich eher als Effekt erfolgreicher medialer Inszenierung eingespielt, wenn Hamburg in kühlen und Konstanz in warmen Farben erscheint, wenn in Köln besonders viele Opfer aus dem Prekariat stammen, in München Stadtteilvielfalt zelebriert und in Kiel gerne getanzt wird? «160 Die einzelnen Beiträge des Sammelbands finden darauf durchaus unterschiedliche Antworten. Es zeigt sich aber, dass Krimis (vielleicht auch

153 Ebd., S. 85,

154 Ebd.

155 Vgl. etwa Guckes, Schürmann 2005; Saldern, Seegers 2005; Guckes 2011.

156 Griem, Scholz 2010, S. 18.

157 Ebd., S. 19.

158 Ebd., S. 20.

159 Ebd., S. 20 f.

160 Ebd., S. 21.

Leviathan, 41. Jg., 2/2013 
andere Genres) vor dem Hintergrund des Eigenlogik-Konzepts neu betrachtet werden können.

»Neue, ergänzende Perspektiven « verspricht der Eigenlogik-Ansatz auch für Stadtplanung und -entwicklung, zuletzt in einem eigens dafür konzipierten Handbuch für die Praxis, das »erstmals die Ergebnisse soziologischer Forschung für die praktische Arbeit an, in und mit Städten « übersetzen soll. ${ }^{161}$ Das Ergebnis dieses Übersetzungsversuchs hat allerdings auch einige kritische Stimmen heraufbeschworen, die monieren, dass die Empfehlungen für die Praxis selten über Allgemeinplätze wie die Forderung nach einer lokalsensiblen Planung, nach signifikanten städtischen Symbolen und breiter Bürgerbeteiligung hinausgingen. Auch die Einsicht, dass nicht jede Entwicklungsstrategie, die in der einen Stadt erfolgreich ist, für eine zweite oder dritte Stadt passen muss, wurde als wenig überraschend kritisiert. Katrin Ecker merkt zudem an, dass die Logiken der Handlungsfelder Wirtschaft, Kultur, Politik und Bauen auf der Suche nach eigenlogischen Einflussfaktoren zu sehr in den Hintergrund treten. ${ }^{162}$ So bleibt die alte Frage, wie sich soziologische Erkenntnisse überhaupt für Stadtplanung und -entwicklung fruchtbar machen lassen, ohne dabei - wie es Häußermann und Siebel 1978 polemisch formuliert haben - zur theorieund positionsarmen »Stadtplanungssoziologie « 163 zu werden, weiterhin unbeantwortet und damit eine Herausforderung für die Zukunft.

\section{Schluss}

Am Beginn dieses Textes stand die simple Frage danach, ob "Stadt « ein eigener Gegenstandsbereich soziologischer Analysen und Theoriebildung sein kann. Stellt man die eigenlogische neben die kritische Stadtsoziologie, erhält man ein »Ja, unbedingt! « und ein »Nein, auf keinen Fall! «. Der Grund für diese gegensätzlichen Antworten ist in unterschiedlichen stadtsoziologischen Konzeptionen zu finden: Während Häußermann und Siebel Stadt und Raum als bloße Erscheinungsformen gesellschaftlicher Strukturen begreifen und deshalb auf die Erforschung der dahinter liegenden gesellschaftlichen Entwicklungen drängen, plädieren Berking und Löw dafür, die städtische Alltagswirklichkeit in ihrem phänomenologischen Reichtum ernst zu nehmen. Die kritische Stadtsoziologie weist der Stadt die Funktion eines »Katalysators «, »Filters « oder » Kompressors « ${ }^{164}$ gesamtgesellschaftlicher Prozesse zu, während im Rahmen des Eigenlogik-Ansatzes der Stadt als soziologischer Gegenstand sui generis neue Aufmerksamkeit zukommt.

Blickt man auf die bisherige Debatte zurück, so fällt ins Auge, dass bislang vor allem die Differenzen der beiden Forschungsperspektiven betont wurden, was zur beständigen Reproduktion eines - häufig polemisch unterlegten - Dualismus zwischen »subsumtionslogischer «/»kritischer « Stadtforschung hier und »eigenlogi-

161 Vgl. Löw, Terizakis 2011.

162 Ecker 2012, S. 59.

163 Vgl. Häußermann, Siebel 1978, S. 491-493.

164 Häußermann, Siebel 2004, S. 100. 
scher «/»kulturalistischer «Stadtforschung dort geführt hat. Ausgeblieben ist in dieser Entgegensetzung eine Auseinandersetzung darüber, welche Annahmen im Rahmen einer wissens- und kultursoziologisch angeleiteten stadtsoziologischen Suchbewegung tatsächlich innovativ und tragfähig sein können, welche einer kritischen Prüfung nicht standhalten und an welchen Stellen Anschlussmöglichkeiten bestehen. Auffällig und erklärungsbedürftig ist zudem, dass in den letzten Jahren zwar intensiv über den Ansatz der Eigenlogik diskutiert worden ist, aber eine Auseinandersetzung mit den Positionen der kritischen Stadtsoziologe bislang weitgehend ausgeblieben ist.

Abschließend stellt sich also einerseits die Frage, warum es nicht verschiedene Ansätze in der (deutschsprachigen) Stadtsoziologie geben kann, die sich gegenseitig ergänzen oder gar befruchten. Andererseits wäre zu fragen, wie beide Herangehensweisen produktiv zusammengedacht werden könnten. Auf diese Fragen eine Antwort zu finden, also sowohl die Perspektive der kulturalistischen Wende und der Hinwendung zum Raum in den Sozial- und Kulturwissenschaften in die stadtsoziologische Wissensproduktion zu integrieren als auch einen kritischen, der Stadt übergeordnete Vergesellschaftungsprozesse systematisch mit bedenkenden Forschungsansatz weiterzuverfolgen, stellt die wohl größte Herausforderung für die Stadtsoziologie in den kommenden Jahren dar. ${ }^{165}$ Eine Annahme dieser Herausforderung verspräche, die deutschsprachige Stadtsoziologie inmitten aktueller (internationaler) soziologischer Debatten um »Gesellschaft ", »Raum « und »das Soziale « zu verorten, an die sie sich in den letzten Jahren - nicht zuletzt wegen des beschriebenen Dualismus - kaum als anschlussfähig erwiesen hat. ${ }^{166}$ Insofern ist dieser Beitrag ein Plädoyer dafür, die bisherige Debatte, die einseitig die Eigenlogik-These umkreist hat, auf die in diesem Beitrag herausgearbeiteten (stadt)soziologischen Grundpositionen der kritischen und eigenlogischen Stadtsoziologie auszuweiten und den darin steckenden Perspektivenstreit, der auch allgemein soziologische Fragen berührt, zu bearbeiten.

\section{Literatur}

Berger, Peter L.; Luckmann, Thomas 2004 [1966]. Die gesellschaftliche Konstruktion der Wirklichkeit: Eine Theorie der Wissenssoziologie. Frankfurt a. M.: Fischer.

Berking, Helmuth 1998. » Global flows and local cultures‘. Über die Rekonfiguration sozialer Räume im Globalisierungsprozeß «, in Berliner Journal für Soziologie 8, 3, S. 381-392.

Berking, Helmuth 2002. "Local frames and global images - nation state and new urban underclass: Über die Globalisierung lokaler Wissensbestände ", in Differenzierungen des Städtischen, hrsg. v. Löw, Martina, S. 107-123. Opladen: Leske + Budrich.

Berking, Helmuth. Hrsg. 2006. Die Macht des Lokalen in einer Welt ohne Grenzen. Frankfurt a. M., New York: Campus.

Berking, Helmuth 2008. " Städte lassen sich an ihrem Gang erkennen wie Menschen - Sizzen zur Erforschung der Stadt und der Städte", in Die Eigenlogik der Städte. Neue Wege für die Stadtforschung, hrsg. v. Berking, Helmuth; Löw, Martina, S. 15-31. Frankfurt a. M., New York: Campus.

165 Vgl. Wietschorke 2012.

166 Vgl. Reckwitz 2009.

Leviathan, 41. Jg., 2/2013 
Berking, Helmuth; Frank, Sybille 2010. "Glasgow's miles better, Dortmund macht sich schön. Stadtinszenierungen im Vergleich «, in Sozialer Sinn 2, S. 163-185.

Berking, Helmuth; Löw, Martina 2005. »Wenn New York nicht Wanne-Eickel ist ... Über Städte als Wissensobjekt der Soziologie ", in Die Wirklichkeit der Städte, hrsg. v. Berking, Helmuth; Löw, Martina, S. 9-22. Baden-Baden: Nomos.

Berking, Helmuth; Löw, Martina. Hrsg. 2008 a. Die Eigenlogik der Städte: Neue Wege für die Stadtforschung. Frankfurt a. M., New York: Campus.

Berking, Helmuth; Löw, Martina 2008 b. »Einleitung «, in Die Eigenlogik der Städte. Neue Wege für die Stadtforschung, hrsg. v. Berking, Helmuth; Löw, Martina, S. 7-14. Frankfurt a. M., New York: Campus.

Berking, Helmuth; Schwenk, Jochen 2011. Hafenstädte. Bremerhaven und Rostock im Wandel. Frankfurt a. M., New York: Campus.

Blanke, Bernhard; Benzler, Susanne; Heinelt, Hubert 1989. »Arbeitslosigkeit im Kreislauf der Politik: eine konzeptionell erweiterte Policy-Analyse zur Erklärung unterschiedlicher Aktivitäten gegen Arbeitslosigkeit auf lokaler Ebene", in Gegenwartskunde 38, 4, S. 529-560.

Bockrath, Franz 2008. »Städtischer Habitus - Habitus der Stadt «, in Die Eigenlogik der Städte. Neue Wege für die Stadtforschung, hrsg. v. Berking, Helmuth; Löw, Martina, S. 55-82. Frankfurt a. M., New York: Campus.

Bürk, Thomas 2011. »Wie der Habitus über die Stadt kam. Erkundungen zur Wanderung und Wandlung des Konzepts >City Habitus«", in Lokalistische Stadtforschung, kulturalisierte Städte. Zur Kritik einer »Eigenlogik der Städte", hrsg. v. Kemper, Jan; Vogelpohl, Anne, S. 137-155. Münster: Westfälisches Dampfboot.

Christmann, Gabriela B. 2011. "Stadtsoziologie", in Soziologische Revue 33, S. 397-407.

Ecker, Katrin 2012. "Städte und ihre Eigenlogik. Ein Handbuch? «, in dérive. Zeitschrift für Stadtforschung 47, S. 59-60.

Frank, Sybille 2012. »Eigenlogik der Städte«, in Handbuch Stadtsoziologie, hrsg. v. Eckardt, Frank, S. 289-309. Wiesbaden: Springer VS.

Gestring, Norbert 2011. »Habitus, Handeln, Stadt - Eine soziologische Kritik der •Eigenlogik der Städte «, in Lokalistische Stadtforschung, kulturalisierte Städte. Zur Kritik einer »Eigenlogik der Städte", hrsg. v. Kemper, Jan; Vogelpohl, Anne, S. 40-53. Münster: Westfälisches Dampfboot.

Giddens, Anthony 1988. Die Konstitution der Gesellschaft. Grundzüge einer Theorie der Strukturierung. Frankfurt a. M., New York: Campus.

Griem, Julika; Scholz, Sebastian. Hrsg. 2010. Tatort Stadt: Mediale Topographien eines Fernsehklassikers. Frankfurt a. M., New York: Campus.

Guckes, Jochen 2011. Konstruktionen bürgerlicher Identität: Städtische Selbstbilder in Freiburg, Dresden und Dortmund 1900-1960. Paderborn: Ferdinand Schöningh.

Guckes, Jochen; Schürmann, Sandra. Hrsg. 2005. Stadtbilder und Stadtrepräsentationen. Themenschwerpunkt der Zeitschrift Informationen zur modernen Stadtgeschichte, Heft $1 / 2005$.

Häußermann, Hartmut 2011. » Soziologie der Städteく. Eine Rezension«, in Lokalistische Stadtforschung, kulturalisierte Städte. Zur Kritik einer »Eigenlogik der Städte", hrsg. v. Kemper, Jan; Vogelpohl, Anne, S. 158-171. Münster: Westfälisches Dampfboot.

Häußermann, Hartmut; Siebel, Walter 1978. »Thesen zur Soziologie der Stadt ", in Leviathan 6, S. 484-500.

Häußermann, Hartmut; Siebel, Walter 1987. Neue Urbanität. Frankfurt a. M.: Suhrkamp.

Häußermann, Hartmut; Siebel, Walter 2004. Stadtsoziologie: Eine Einführung. Frankfurt a. M., New York: Campus.

Held, Gerd 2005. Territorium und Großstadt. Die räumliche Differenzierung der Moderne. Wiesbaden: VS Verlag für Sozialwissenschaften.

Herrmann, Heike et al. Hrsg. 2001. Die Besonderheit des Städtischen: Entwicklungslinie der Stadt(soziologie). Wiesbaden: VS Verlag für Sozialwissenschaften.

Höhne, Stefan 2011. »Identitätslogische Prämissen des eigenlogischen Denkens und die Multiplikatoren des Urbanen ", in Lokalistische Stadtforschung, kulturalisierte Städte. Zur Kritik einer »Eigenlogik der Städte", hrsg. v. Kemper, Jan; Vogelpohl, Anne, S. 54-72. Münster: Westfälisches Dampfboot.

Kastner, Jens 2009 a. »Löw, Martina, Soziologie der Städte«, in Das Argument 280, S. 352-353. 
Kastner, Jens 2009 b: "Städtische Eigenlogiken «, in dérive. Zeitschrift für Stadtforschung 34, S. 54.

Kemper, Jan; Vogelpohl, Anne 2011. » Eigenlogik der Städte?‘. Kritische Anmerkungen zu einer Forschungsperspektive", in Lokalistische Stadtforschung, kulturalisierte Städte. Zur Kritik einer "Eigenlogik der Städte", hrsg. v. Kemper, Jan; Vogelpohl, Anne, S. 15-38, Münster: Westfälisches Dampfboot.

Krämer-Badoni, Thomas 1991. »Die Stadt als sozialwissenschaftlicher Gegenstand «, in Stadt und Raum: Soziologische Analysen, hrsg. v. Häußermann, Hartmut et al., S. 1-29. Pfaffenweiler: Centaurus.

Krämer-Badoni, Thomas 2011. „Die Klassiker der Stadtsoziologie und die Besonderheit des Städtischen ", in Die Besonderheit des Städtischen: Entwicklungslinie der Stadt(soziologie), hrsg. v. Herrmann, Heike et al., S. 69-83. Wiesbaden: VS Verlag für Sozialwissenschaften.

Lee, Martyn 1997. "Relocating location: cultural geography, the specificity of place and the city habitus ", in Cultural methodologies, hrsg. v. McGuigan, Jim, S. 126-141. London et al.: Sage.

Lindner, Rolf 2003. »Der Habitus der Stadt. Ein kulturgeographischer Versuch «, in Petermanns Geographische Mitteilungen 147, S. 46-53.

Lindner, Rolf 2005 a. "Die Kultur der Metropole", in Humboldt Spektrum 12, 2, S. 22-28.

Lindner, Rolf 2005 b. »Urban anthropology «, in Die Wirklichkeit der Städte, hrsg. v. Berking, Helmuth; Löw, Martina, S. 55-66. Baden-Baden: Nomos.

Lindner, Rolf 2008. »Textur, imaginaire, Habitus - Schlüsselbegriffe der kulturanalystischen Stadtforschung ", in Die Eigenlogik der Städte. Neue Wege für die Stadtforschung, hrsg. v. Berking, Helmuth; Löw, Martina, S. 83-94. Frankfurt a. M., New York: Campus.

Lindner, Rolf; Moser, Johannes. Hrsg. 2006. Dresden. Ethnografische Erkundungen einer Residenzstadt. Leipzig: Leipziger Universitätsverlag.

Löw, Martina 2001. Raumsoziologie. Frankfurt a. M.: Suhrkamp.

Löw, Martina 2008 a. »Eigenlogische Strukturen - Differenzierungen zwischen Städten als konzeptionelle Herausforderungen ", in Die Eigenlogik der Städte. Neue Wege für die Stadtforschung, hrsg. v. Berking, Helmuth; Löw, Martina, S. 33-53. Frankfurt a. M., New York: Campus.

Löw, Martina 2008 b. Soziologie der Städte. Frankfurt a. M.: Suhrkamp.

Löw, Martina 2011. "Städte als sich unterscheidende Erfahrungsräume: Grundlagen für eine sinnverstehende Stadtsoziologie ", in Die Besonderheit des Städtischen: Entwicklungslinie der Stadt(soziologie), hrsg. v. Herrmann, Heike et al., S. 49-67. Wiesbaden: VS Verlag für Sozialwissenschaften.

Löw, Martina; Terizakis, Georgios. Hrsg. 2011. Städte und ibre Eigenlogik. Ein Handbuch für Stadtplanung und Stadtentwicklung. Frankfurt a. M., New York: Campus.

Marx, Karl; Engels, Friedrich 1969 [1932]. Werke, hrsg. v. Institut für Marxismus-Leninismus beim ZK der SED, Bd. 3. Berlin: Dietz.

Matthiesen, Ulf 1997. "Lebensweltliches Hintergrundwissen " in Konfigurationen lebensweltlicher Strukturphänomene, hrsg. v. Wicke, Michael, S. 157-178. Opladen: Leske + Budrich.

Michel, Boris 2009. »Berking, Helmuth u. Martina Löw (Hrsg.), Die Eigenlogik der Städte. Neue Wege für die Stadtsoziologie ", in Das Argument 280, S. 353-354.

Michel, Boris 2011. »Neue Wege in die Stadtforschung oder fragwürdige Abstraktionen? Zur reigenlogischen< Lesart der >Neuen Stadtsoziologie'", in Lokalistische Stadtforschung, kulturalisierte Städte. Zur Kritik einer »Eigenlogik der Städte ", hrsg. v. Kemper, Jan; Vogelpohl, Anne, S. 118-136. Münster: Westfälisches Dampfboot.

Musner, Lutz 2009. Der Geschmack von Wien. Kultur und Habitus einer Stadt. Frankfurt a. M., New York: Campus.

Reckwitz, Andreas 2009. "Die Selbstkulturalisierung der Stadt «, in Mittelweg 36 2, S. 2-34.

Saldern, Adelheid von; Seegers, Lu. Hrsg. 2005. Inszenierter Stolz: Stadtrepräsentationen in drei deutschen Gesellschaften (1935-1975). Stuttgart: Steiner.

Schott, Dieter 2012. »'Eigenlogik der Städte`: Abkehr von der Urbanisierungsforschung? «, in Informationen zur modernen Stadtgeschichte 2, S. 76-86.

Schroer, Markus 2006. Räume, Orte, Grenzen. Auf dem Weg zu einer Soziologie des Raumes. Frankfurt a. M.: Suhrkamp. 
Schütz, Alfred 1974. Der sinnhafte Aufbau der sozialen Welt: Eine Einleitung in die verstehende Soziologie. Frankfurt a. M.: Suhrkamp.

Sept, Ariane 2009. »Helmuth Berking, Martina Löw (Hrsg.): Die Eigenlogik der Städte «, in RaumPlanung 146, S. 247-248.

Siebel, Walter 2012. "Stadt und soziale Ungleichheit", in Leviathan 40, 3, S. 462-475.

Simmel, Georg 1957 [1903]. »Die Großstädte und das Geistesleben «, in Georg Simmel: Brücke und Tür. Essays des Philosophen zur Geschichte, Religion, Kunst und Gesellschaft, hrsg. v. Landmann, Michael; Susman, Margarete, S. 227-242. Stuttgart: Köhler.

Strauss, Anselm L. 1961. Images of the American city. New York: Free Press of Glencoe.

Suttles, Gerald D. 1984. »The cumulative texture of local urban culture «, in American Journal of Sociology 90, 2, S. 283-304.

Thomas, William I. 1928. The child in America: behavior problems and programs. New York: Alfred A. Knopf.

Wietschorke, Jens 2009. »Rezension zu: Löw, Martina: Soziologie der Städte. Frankfurt a. M. 2008 ", in H-Soz-u-Kult. http://hsozkult.geschichte.hu-berlin.de/rezensionen/2009-4-048 (Zugriff vom 04.12.2012).

Wietschorke, Jens 2012. »Rezension zu: Kemper, Jan; Vogelpohl, Anne (Hrsg.): Lokalistische Stadtforschung, kulturalisierte Städte. Zur Kritik einer >Eigenlogik der Städte‘. Münster 2011 ", in $H$-Soz-u-Kult. http://hsozkult.geschichte.hu-berlin.de/rezensionen/2012-2-043 (Zugriff vom 04.12.2012).

Wirth, Louis 1974 [1938]. »Urbanität als Lebensform «, in Stadt und Sozialstruktur. Arbeiten zur sozialen Segregation, Ghettobildung und Stadtplanung, hrsg. v. Herlyn, Ulfert, S. 42-66. München: Nymphenburger.

Wohl, Richard R.; Strauss, Anselm 1958. »Symbolic representation and the urban milieu «, in American Journal of Sociology 63, 5, S. 523-532.

Zimmermann, Karsten 2012. »Organisationsfelder, Arenafärbungen und städtische Eigenlogiken. Einige konzeptuelle Überlegungen zum Repertoire lokaler Politikforschung ", in Regieren. Festschrift für Hubert Heinelt, hrsg. v. Egner, Björn; Haus, Michael; Terizakis, Georgios, S. 281-295. Wiesbaden: Springer VS. 
Zusammenfassung: Kann »Stadt « ein Gegenstand soziologischer Analysen und Theoriebildung sein? Über diese Frage streitet derzeit die deutschsprachige Stadtsoziologie. Der vorliegende Beitrag strukturiert diese Debatte und plädiert dafür, den ihr zugrunde liegenden Perspektivenstreit zwischen einem kritischen und einem wissens- und kulturbasierten stadtsoziologischen Ansatz für allgemein soziologische Fragen zu öffnen.

Stichworte: Stadtsoziologie, kritische Stadtsoziologie, Eigenlogik, Debatte, Stadt, Soziologie

\section{The current conceptual debate in urban sociology}

Summary: Can »city « be a subject of sociological analyses and theory-building? This paper introduces an ongoing debate in German urban sociology that revolves around this question. After structuring the competing concepts of a critical and a knowledge-based sociological perspective on cities, this paper proposes shifting the focus towards the fundamental sociological questions underlying this debate.

Keywords: urban sociology, critical urban sociology, Eigenlogik, intrinsic logic, debate, city, sociology

\section{Autorinnen und Autoren}

Prof. Dr. Sybille Frank

Institut für Soziologie

Technische Universität Berlin

Fraunhoferstraße 33-36

10587 Berlin

sybille.frank@tu-berlin.de

Jochen Schwenk M.A.

Institut für Soziologie

Technische Universität Darmstadt

Residenzschloss

64283 Darmstadt

schwenk@ifs.tu-darmstadt.de

Dr. Silke Steets

Institut für Soziologie

Technische Universität Darmstadt

Residenzschloss

64283 Darmstadt

steets@ifs.tu-darmstadt.de

Dipl.-Soz. Gunter Weidenhaus

Institut für Soziologie

Technische Universität Darmstadt

Residenzschloss

64283 Darmstadt

weidenhaus@ifs.tu-darmstadt.de

Leviathan, 41. Jg., 2/2013 